NOTA DE INVESTIGACION

\title{
CONTROL DEL MINADOR DE LAS HOJAS DE LOS CÍTRICOS (Phyllocnistis citrella) EN PLANTAS JÓVENES DE NARANJA VALENCIA
}

\author{
Bertuzzi, Silvia M.; Rodriguez, Víctor A.; Mazza, Silvia M. \\ Facultad de Ciencias Agrarias - Universidad Nacional del Nordeste \\ Sargento Cabral 2131 - (3400) CORRIENTES. \\ Trabajo financiado por la Secretaría General de Ciencia y Técnica de la UNNE.
}

\section{RESUMEN}

El minador de las hojas de los cítricos (Phyllocnistis citrella, Stainton), ataca los brotes tiernos de las plantas destruyéndolos por completo. Debido a su aparición en el Nordeste Argentino, resulta necesario ajustar técnicas de control. Con el objetivo de determinar el comportamiento y residualidad de diferentes formas de aplicación de Imidacloprid y su comparación con abamectin para su control, entre 1996 y 1998, se llevó a cabo una experiencia en plantas de naranja Valencia (Citrus sinensis) a partir de los 2 años de implantadas. Los tratamientos fueron: 1) Testigo; 2) Imidacloprid $35 \%$ al $0,05 \%, 5$ I de solución por m de altura de planta, aplicado al suelo; 3 ) Imidacloprid $35 \%$,

al $0,1 \%, 5$ I por $\mathrm{m}$ de altura, aplicado al suelo; 4) Imidacloprid $35 \%$ al $0,02 \%$ más aceite $0,5 \%$, pulverización foliar y 5) Abamectin 0,025\% más aceite $0,5 \%$, pulverización foliar. El diseño fue totalmente aleatorizado con 4 repeticiones, parcela útil 1 planta y sus respectivas borduras. Los trabajos se iniciaron en diciembre de 1996, determinando cada 15 días el porcentaje de brotes sanos entre diciembre y abril de cada año. Las plantas testigo mantuvieron siempre entre 20 y $30 \%$ de brotes sanos. Los tratamientos 4 y 5 (pulverizaciones foliares), requirieron tres aplicaciones para mantener los brotes con bajos niveles de ataque; en los tratamientos 2 y 3 (en suelo), la aplicación inicial mantuvo los brotes de las plantas libres de larvas durante toda la evaluación.

Palabras clave: imidacloprid, control fitosanitario, insecticidas.

\section{SUMMARY}

The citrus leaf miner (Phyllocnistis citrella, Stainton) is a pest that attacks the new flush of trees, destroying them completely. In the Northeastern of Argentine its appearance was recent, this created the necesity of adjustment and evaluation of the control techniques for this pest. With the objective of determining the behavior and residuality of different forms of imidacloprid application and its comparison with abamectin in the leaf miner control, an experience with two years old Valencia Late orange was made between 1996 and 1998. The treatments tested were: 1) Control; 2) Imidacloprid $35 \%$ at $0.05 \%$ solution on with 5 I application for meter of plant height; 3) Imidacloprid $35 \%$, at $0.1 \%$ solution with 51 for meter of plant height.; 4) Imidacloprid $35 \%, 0.02 \%$ plus $0.5 \%$ oil and 5 ) Abamectin $0.025 \%$ plus $0.5 \%$ oil. Both treatments, 2 and 3, were soil application and both treatments, 4 and 5 , were with foliar pulverization. The statistical design was completely randomized with four replications and a useful plot of one plant with its borders. Field works began in December ' 96 . Every fifteen days until April the percent of healthy buds was determined. Control plants had between 20 and $30 \%$ of healthy leaves per flush. In treatments 4 and 5 three applications were necessary to maintain low attack level in young leaves; in treatments 2 and 3 (soil application) the initial application was enough to keep the young leaves free from leaf miner during all the evaluation time.

Key words: imidacloprid; phitosanitary control; insecticide. 


\section{ANTECEDENTES}

Phyllocnistis citrella Stainton, es un Lepidóptero perteneciente a la familia Gracillariidae, Subfamilia Phylocnistinae (Clausen, 1981), comúnmente se conoce con el nombre de minador de las hojas de los cítricos. Es una especie originaria del Sudeste Asiático, constituyéndose en una plaga de gran importancia para las plantaciones de China, India y Japón. Posteriormente se expandió a Australia e Islas del Pacífico, quedando confinada a estas regiones hasta 1986 (CAB). En 1993 se detectó en Florida (Knapp. et al, 1993) y casi simultáneamente en la Cuenca del Mediterráneo (Amorós. et al, 1994, y Cayetano et al, 1994). En España se lo observó por primera vez en Málaga (Garijo et al, 1994). En Argentina este insecto fue detectado a fines del otoño de 1996 en plantaciones de Salta, Jujuy y Tucumán (J. Palacios, comunicación personal); en el Nordeste Argentino (NEA), en plantaciones de Bella Vista y zonas de influencia fue observado por primera vez en noviembre del mismo año (S. Cáceres, comunicación personal), se registró como plaga en forma oficial en Corrientes el 18 de noviembre de 1996.

Este fitófago, se alimenta preferentemente de especies pertenecientes a la familia de las Rutáceas, aunque también se lo puede encontrar sobre diferentes especies de Leguminosae y Proteaceae (Balachowski, 1966). Los daños lo efectúan las larvas en brotes y pequeñas hojas, al separar la cutícula del parénquima produciendo galerias o minas sinuosas sobre el mesófilo. La cutícula se rompe con facilidad originando el desecamiento y caida de las hojas y la inactivación total de aquellos brotes que permanecen en las plantas.

En plantaciones jóvenes, los daños producidos por este lepidóptero adquieren singular importancia ya que las pequeñas plantas se verán imposibilitadas de crecer y desarrollarse, por lo que se puede ver comprometida la formación del árbol y la pronta entrada en producción. De allí la importancia del control en las sucesivas brotaciones que se produzcan.

Rojo Sánchez et al. (1997), en España trabajaron sobre control del minador en plantaciones de naranjo var. Lane Late, injertados sobre mandarino Cleopatra, de un año de implantados, probando aspersión foliar de abamectin con aceite mineral, aplicaciones al suelo de aldicarb e imidacloprid en riego por goteo y pintado al tronco, encontraron que imidacloprid en las dos formas de aplicación y aldicarb otorgaron buena protección a las plantas; abamectin, en cambio, dio una protección solamente aceptable.

En plantaciones de clemenules de dos y tres años, injertados sobre híbridos de trifolio Citrange Carrizo, Ripollés et al. (1996), probaron diferentes tipos de aceites minerales en dosis de 0,5 y $1 \%$ y su comparación con imidacloprid al $0.015 \%$ para el control de $P$. citrella, encontraron los mejores resultados con imidacloprid solo y con aceites al $1 \%$, aunque en con efecto de fitotoxicidad cuando las pulverizaciones de aceite en esa concentración, excedían el número de cuatro en el período estival.

Martinez Ferrer et al (2000), en España, probaron diferentes aceites emulsionables al $1 \%$ y consiguieron más de un $70 \%$ de control en cuatro aplicaciones.

Bullock y Pelosi (1996), trabajando en experimentos a campo demostraron la eficacia del imidacloprid en el control de $P$. citrella, pintando el tallo de las plantas a razón de $1 \mathrm{ml}$ de producto formulado por $\mathrm{cm}$ de diámetro.

Ante la detección de $P$. citrella en el NEA, este trabajo se realizó con el objetivo de determinar el comportamiento y residualidad de diferentes formas de aplicación de imidacloprid y su comparación con abamectin, en el control del minador de las hojas en plantas jóvenes de Citrus.

\section{MATERIALES Y MÉTODOS}

El trabajo se efectuó en el Campo Experimental de la Facultad de Ciencias Agrarias (FCA), Universidad Nacional del Nordeste (UNNE), Corrientes, en plantas de naranja (Citrus sinensis, Osbeck) var. Valencia de 3 años de edad, injertadas sobre lima Rangpur (Citrus limonia, Osbeck) e implantadas en un suelo Udipsamment álfico a una distancia de 7 por 3.5 (408 plantas por hectárea).

Los tratamientos probados fueron: 1) testigo; 2) imidacloprid (Confidor $35 \%$ comercializado por BAYER Argentina S.A.) al 0,05\%, 5 litros de solución por $\mathrm{m}$ de altura de planta, aplicado al suelo como riego en zona de raíces; 3 ) imidacloprid al $0,1 \%, 5$ litros por $\mathrm{m}$ altura, aplicado al suelo de igual forma al tratamiento anterior; 4) imidacloprid a $0,02 \%$ más aceite al $0,5 \%$, en pulverización foliar, a razón de 1,5 litro de solución por planta y 5 ) abamectin (Vertimec $1.8 \%$, comercializado por Novartis) en concentración del $0,025 \%$ más aceite al $0,5 \%$, en pulverización foliar, a razón de 1,5 litros de solución por planta. Los trabajos se efectuaron entre los meses de diciembre de 1996 y abril de 
1998. La primer aplicación anual para todos los tratamientos se efectuó sobre la brotación de diciembre de cada año, mientras que los tratamientos 4 y 5 fueron repetidos durante cada brotación de verano.

El diseño empleado fue totalmente aleatorizado, con 4 repeticiones. La parcela útil estaba conformada por una planta, con sus respectivas borduras. Entre los meses de diciembre y abril de cada año, aproximadamente, cada 15 días se determinó por ciento de brotes sanos de hasta 2 $\mathrm{cm}$. de longitud. Para cada evaluación se efectuó el análisis de la variancia y prueba de Tukey, previa transformación de los por cientos de brotes sanos mediante la raíz cuadrada de $x+0.5$. Los análisis se efectuaron mediante Statgraphics 6.0 .

\section{RESULTADOS Y DISCUSIÓN}

En la evaluación previa a la aplicación de los tratamientos (Evaluación 1), no se detectaron diferencias significativas entre estos, lo que asegura una infestación homogénea entre las parcelas seleccionadas para la aplicación de cada uno de ellos.

La Tabla 1 presenta los porcentajes de brotes sanos, en promedio para las cuatro repeticiones de cada tratamiento y en el conjunto de los años, en cada evaluación y los resultados del análisis estadístico por el período bajo estudio.

En las evaluaciones post-tratamiento, hasta la sexta, todos los tratamientos con aplicación se diferencian significativamente del testigo, a un nivel de significación de $\alpha=0,01$. En la evaluación 7, los tratamientos 2 a 5 se diferencian del testigo, los tratamientos 2 y 3 se diferencian de 4 y $5(\alpha=0,01)$. En la evaluación 8 , no se realizó el análisis estadístico ya que solamente el testigo presentó brotación.

Los tratamientos con imidacloprid aplicado al suelo, en zona de raíces, al $0,05 \%$ y al $0,10 \%$ mantuvieron totalmente libres de daños del insecto las brotaciones producidas en fines de enero, febrero y marzo de cada año, por lo que no fueron necesarias nuevas aplicaciones.

Los tratamientos con imidacloprid aplicado en pulverización foliar $y$ abamectin tuvieron un comportamiento similar $y$, para mantener los brotes en condiciones adecuadas que aseguraran una normal actividad fotosintética, requirieron en total tres aplicaciones $y$ aún así, las últimas brotaciones de marzo presentaron un daño promedio del $40 \%$ en las cuatro repeticiones y en el conjunto de los años.

En todas las parcelas tratadas no se produjeron nuevas brotaciones a partir de abril, sin embargo en las parcelas testigo las brotaciones fueron permanentes, sin que las plantas entraran en reposo hasta el pleno inviemo. Los porcentajes de brotes sin larvas de minador oscilaron en todo momento entre 10 y 30 , esto sin duda agrava el efecto de daños de esta plaga, ya que al destruir permanentemente los sucesivos brotes de las plantas jóvenes las debilitan considerablemente comprometiendo su futura productividad.

Tabla 1: Porcientos de brotes de naranja sin ataque de minador, promedio de cuatro repeticiones en el conjunto de los años, en las diferentes evaluaciones efectuadas ${ }^{(\mathrm{Z})}$ :

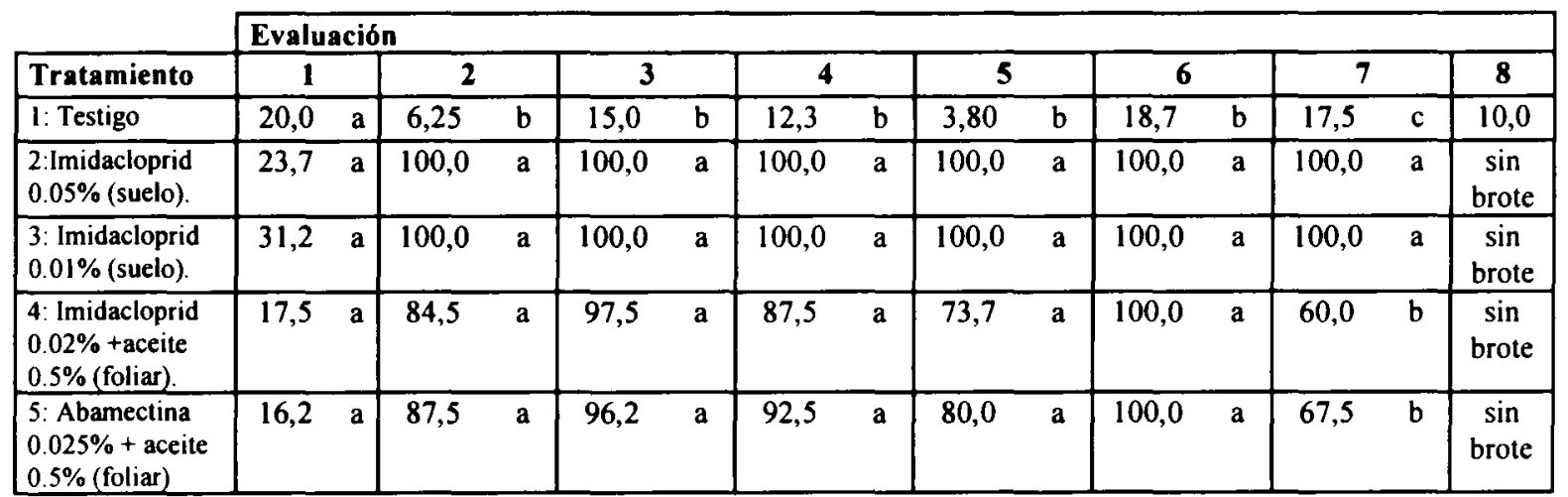

${ }^{(\mathrm{Z})}$ Letras diferentes en las columnas indican promedios estadísticamente diferentes, según Tukey ( $\alpha \leq$ $0,01)$. 


\section{CONCLUSIONES}

Conforme a los resultados obtenidos, en condiciones similares a las que se llevó a cabo esta experiencia, es posible mantener las plantas libres de ataque de Phyllocnistis citrella con aplicaciones al suelo de imidacloprid al $0.05 \%$ durante más de 120 días.

\section{BIBLIOGRAFÍA}

Amorós, J. y J. Gonell. 1994. Una nueva plaga de la citricultura: el denominado "minador de los cítricos" (Phyllocnistis citrella, Stainton). Levante Agrícola. España. 33(326:77-79).

Balachowski, A.S. 1966. Entomologie Appliquée a l'Agriculture. Tome II. Lepidopteres. Premier Volume. Masson et Cie. $1056 \mathrm{pp}$.

Bullock, R.C. y R.R. Pelosi. 1996. Efficacy of imidacloprid against citrus leafminer (CLM), Phyllocnistis citrella, Stainton, in Florida, USA. In: Actas VIII Congress of the International Society of Citriculture. South Africa. p 47.

Cayetano, A. y J. Garcia. 1994. Minador de la hoja de los citricos, Phyllocnistis citrella, Stainton. Phytoma. España. 58(56-62).

Clausen, C.P. 1981. Two citrus Leaf Miners of the For East. Technical Bulletin $\mathrm{N}^{\circ} 252$. United States Department of Agriculture, Washington, O.C. 1-5.

Commonwealth Agriculture Bureaux (CAB). Commonwealth Institute of Entomology. 1970. Phyllocnistis citrella, Stan. In. Distribution Maps. of Pests. Ser. A. Map. $N^{\circ} 275$. The Eastern Press Ltd. London.
Garijo, C. y R. Castillo. 1994. El minador de las hojas de los cítricos (Phyllocnistis citrella, Stainton). Divulgación Sanidad Vegetal. 0/94. Junta de Andalucía. Consellería de Agricultura y Pesca, Dirección General de Investigación, Tecnología y Formación Agroalimentaria y Pesquera. Sevilla. 8 pp.

Knapp, H.; J.J. Peña; P. Stansly; J. Heppner y Y. Yang. 1993. Citrus leafminer a new pest of citrus in Florida. Citrus Industry. October 1993. pp. 42-43, 62.

Martinez Ferrer, M. T., J. M. Fibla, J. M. Campos y E. Beltrán. 2000. "Using insecticide mineral Oils for controlling Phyllocnistis citrella, Stainton (Lepidoptera : Gracillariedae) and other summer pests". in An Integrated Pest Management Program in Citrus Groves. ISC Congress 2000 Abstract Florida EEUU, p:56.

Ripollés, J.L.; A. Urbaneja y J.Avilla. 1996. Utilización de aceites minerales para el control de Phyllocnistis citrella, Stainton (Lepidoptera : Gracillariedae): el minador de las hojas de los cítricos. Levante Agrícola. España. 33(335:154157).

Rojo Sánchez, J.; J. Jiménez Luque y J.A. García Carmona. 1997. Control del minador de las hojas (Phyllocnistis citrella, Stainton) en plantones de citricos. Levante Agricola. España. 36(338:10-17).

Steel, R.G.D y J.H. Torrie. 1992. Bioestadística, Principios y Procedimientos. Mc Graw Hill. México. pp. 132-185.

\section{Nota editorial}

Las opiniones vertidas por los distintos autores son de su exclusiva responsabilidad.

Las normas generales para la presentación de trabajos están publicadas en Agrotecnia $\mathbf{N}^{\circ} \mathbf{6}$, pág. 19 (2000). También las puede solicitar al Comité Editorial a: tomeice@agr.unne.edu.ar O a la dirección postal Sargento Cabral 2131-3400 Corrientes. 